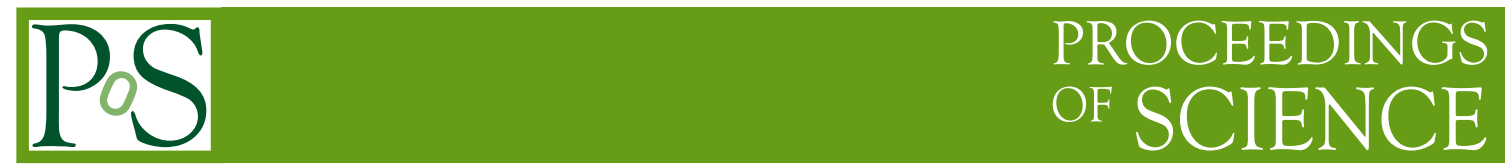

\title{
SFXC: The new EVN data processor
}

\author{
Mark Kettenis* \\ JIVE \\ E-mail: kettenisejive.nl
}

\section{Aard Keimpema}

JIVE

E-mail: keimpema@jive.nl

Since the last EVN symposium the SFXC software correlator has taken over all correlation duties from the venerable Mark4 data processor as the main EVN correlator. Due to its flexible nature it offers many new observation modes. This paper presents the most important new capabilities that SFXC provides and some recent developments.

12th European VLBI Network Symposium and Users Meeting,

7-10 October 2014

Cagliari, Italy

${ }^{*}$ Speaker. 


\section{Introduction}

The SFXC software correlator has now completely replaced the EVN Mark4 correlator at JIVE. Its flexible design offers EVN users many more observing modes than the Mark4 hardware correlator could provide. Key features include:

- virtually unlimited spectral and time resolution

- higher accuracy in fringe rotation and delay tracking

- overlapping FFTs with user-selectable window function (Hann, Hamming, Cosine, ...)

- CALC10-based model (same as Mark4 correlator)

- global VLBI at $1 \mathrm{Gbit} / \mathrm{s}$ and beyond

- Pulsar Gating \& Binning

- Multiple Phase Centers

- Phased Array Mode

Even for traditional continuum and spectral line VLBI observations, the software correlator removes many of the limits of the Mark4 correlator, such as the maximum number of stations, maximum spectral resolution and limits on integration times. This paper describes the, from a user's standpoint, most important features and some recent developments. A more in-depth discussion of SFXC's algorithms and implementation can be found in [1].

\section{Wide-Field VLBI in Smaller Bites}

The field of view of a VLBI observation is typically limited by time and bandwidth smearing. To image the full field of view high spectral and time resolution are needed, resulting in enormous data sets. As an alternative to imaging the whole field of view, the Multiple Phase Center feature of SFXC allows the specification of multiple correlation centers. In this approach, pioneered by Deller et. al. [2], the correlator uses high time and spectral resolution internally. Results are then phase shifted to each specified correlation center and averaged down in time and frequency, resulting in data sets of manageable size. The computational overhead per additional phase center is very low. See figure 1.

\section{Pulsar Gating \& Binning}

With pulsar gating, correlation results are only accumulated during the "on" phase of the pulsar period. This can increase the $\mathrm{S} / \mathrm{N}$ of pulsar observations significantly. With pulsar binning the pulsar period is divided in multiple bins in which correlation results are accumulated. Pulsar binning can be used to create pulse profiles. SFXC can combine gating and binning (see figure 2. In order to use pulsar gating \& binning, PI's need to provide tempo2 polyco files in center-ofearth frame. SFXC supports both incoherent and coherent de-dispersion. Coherent de-dispersion is computationally more expensive but needed for observing high-DM millisecond pulsars at low frequencies. 


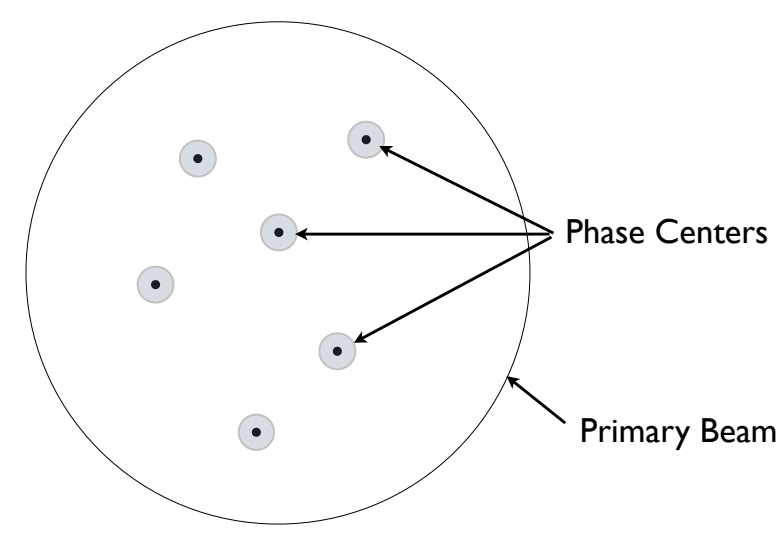

Figure 1: SFXC can correlate multiple simultaneous phase centers within the primary beam of the telescopes of the EVN.
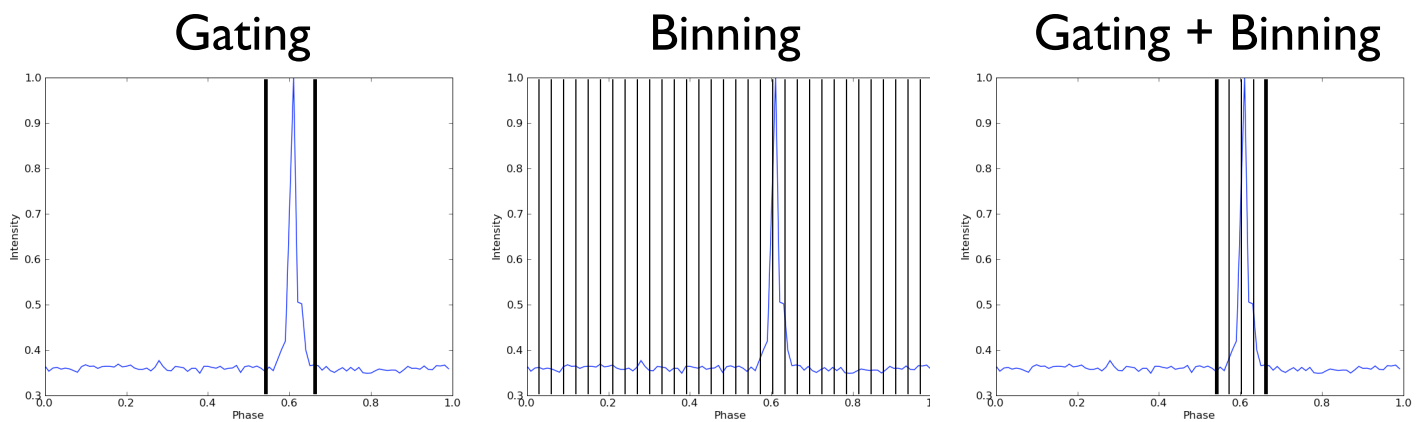

Figure 2: Pulsar gating, pulsar binning and the combination of the two.

\section{Phased Array Mode}

SFXC can be used to phase up VLBI telescopes. This requires a two-pass correlation scheme. After a normal correlation pass, and calibration in AIPS, calibration information is used to coherently add the signals and write PSR-FITS. The output can then be used by standard pulsar tools. For example, we have successfully used PRESTO to "search" for pulsars in EVN observations.

\section{Calibration}

SFXC now includes support for continuous $T_{s y s}$ extraction, providing frequent $T_{s y s}$ measurements while "on-source". It is already in use for VLBA stations in global observations and Effelsberg. This feature will become available for other EVN telescopes when they implement the necessary hardware, Figure 3 shows an example of the measured $T_{s y s}$ for Effelsberg in a recent user experiment. 

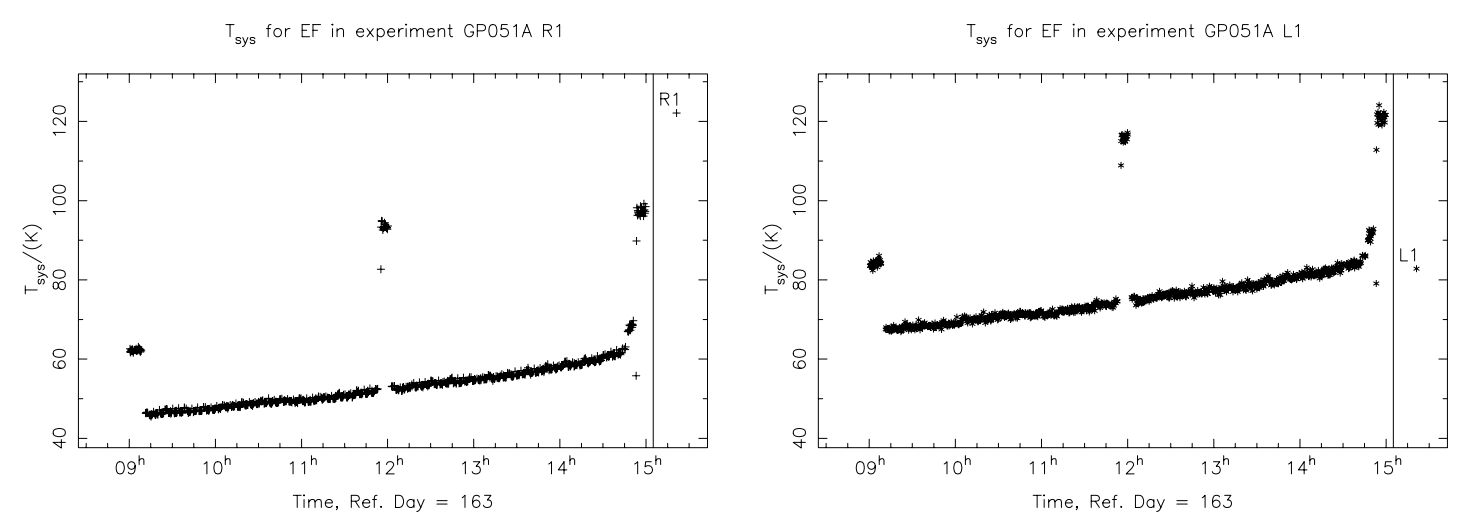

Figure 3: $T_{\text {sys }}$ measurements extracted by SFXC from the baseband data for Effelsberg in GP051A.

\section{Space Science}

By default, SFXC uses the same CALC10-based delay model as the EVN Mark4 correlator. For observations of sources in the solar system a near-field model is required. SFXC doesn't include such a delay model but does implement a "Bring Your Own Model" mode with a possibility to include additional phase and amplitude corrections in addition to simple delays. These additional corrections can be used to do proper Doppler tracking of spacecraft.

\section{Hardware}

The SFXC software runs on a cluster of standard Linux servers. The cluster at JIVE consists of 40 nodes with a total of 384 cores. This is enough hardware to do real-time e-VLBI correlation of 13 stations at $1 \mathrm{Gbit} / \mathrm{s}$ in standard continuum mode, with enough spare capacity to allow for some hardware failures. For traditional disk-based VLBI there are no limitations on the number of stations or the data rate.

\section{Conclusion}

The replacement of the Mark4 correlator with the SFXC software correlator at JIVE offers EVN users many new possibilities. The flexibility of the software approach allows for rapid development of improvements and new features. Development continues, and we hope to present many new features at the next EVN Symposium.

\section{References}

[1] A. Keimpema, M.M. Kettenis, S.V. Pogrebenko et. al. The SFXC software correlator for Very Long Baseline Interferometry: Algorithms and Implementation, submitted to Experimental Astronomy

[2] A.T. Deller et. al. DiFX-2: A More Flexible, Efficient, Robust, and Powerful Software Correlator, PASP 123:275-287, 1101.0885 